\title{
High-Sensitivity Goos-Hänchen Shifts Sensor Based on BlueP-TMDCs-Graphene Heterostructure
}

\author{
Lei Han, Zhimin Hu, Jianxing Pan, Tianye Huang $₫$ and Dapeng Luo * \\ School of Mechanical Engineering and Electronic Information, China University of Geosciences (Wuhan), \\ Wuhan 430074, China; hanlei@cug.edu.cn (L.H.); huzhimin@cug.edu.cn (Z.H.); jianxing_pan@163.com (J.P.); \\ tianye_huang@163.com (T.H.) \\ * Correspondence: luodapeng@cug.edu.cn
}

Received: 29 May 2020; Accepted: 24 June 2020; Published: 26 June 2020

\begin{abstract}
Surface plasmon resonance (SPR) with two-dimensional (2D) materials is proposed to enhance the sensitivity of sensors. A novel Goos-Hänchen (GH) shift sensing scheme based on blue phosphorene (BlueP)/transition metal dichalogenides (TMDCs) and graphene structure is proposed. The significantly enhanced GH shift is obtained by optimizing the layers of BlueP/TMDCs and graphene. The maximum GH shift of the hybrid structure of Ag-Indium tin oxide (ITO)-BlueP/ $/ \mathrm{WS}_{2}-$ graphene is $-2361 \lambda$ with BlueP/ $/ \mathrm{WS}_{2}$ four layers and a graphene monolayer. Furthermore, the GH shift can be positive or negative depending on the layer number of BlueP/TMDCs and graphene. For sensing performance, the highest sensitivity of $2.767 \times 10^{7} \lambda /$ RIU is realized, which is 5152.7 times higher than the traditional Ag-SPR structure, 2470.5 times of Ag-ITO, 2159.2 times of $\mathrm{Ag}$-ITO-BlueP/WS 2 , and 688.9 times of Ag-ITO-graphene. Therefore, such configuration with GH shift can be used in various chemical, biomedical and optical sensing fields.
\end{abstract}

Keywords: surface plasmon resonance; Goos-Hänchen shifts; blue phosphorene; transition metal dichalcogenides; graphene; sensitivity

\section{Introduction}

The Goos-Hänchen $(\mathrm{GH})$ shift refers to the lateral spatial shift of the center of mass of the bounded beam relative to the geometric prediction [1]. The GH migration results from the role dispersion of the Fresnel reflection coefficient [2,3]. When the phase of reflection coefficient changes significantly near the critical angle of total reflection, GH effect can be enhanced [4-6]. Due to the advantages of GH shift in precision measurement and optical sensing, new attention has been observed [7-9]. In the fields of optics, chemistry and sensors, GH shift has been widely discussed $[10,11]$ and there is always a goal for researchers to obtain large GH shifts.

The GH shift at the interface of two homogeneous materials with different optical properties is usually very small, almost equal to the incident wavelength [12]. Leveraging on metal to excite surface plasmon polaritons (SPP) is an effective way to improve GH shift [13]. SPPs are kinds of vertically constrained evanescent electromagnetic waves $[14,15]$. According to Snell's law, if the incident angle is larger than the total reflection angle, the total reflection phenomenon will appear when a beam of light is transmitted from a dense medium to a sparse medium [16]. When total reflection occurs, if there is no energy loss, it is called total internal reflection (TR), if there is energy loss, it is called attenuated total reflection (ATR). From the point of view of physical optics, a more in-depth study of total reflection shows that when total reflection occurs, the beam enters a wavelength-level depth in the optical medium, and its amplitude decays exponentially along the direction perpendicular to the interface. At the same time, in the incident plane, it transmits for a certain distance along the interface direction, and then returns to the optical dense medium. From the point of view of physical 
optics, a more in-depth study of total reflection shows that when total reflection occurs, the beam enters a wavelength level depth in the optical medium, and its amplitude decays exponentially along the direction perpendicular to the interface. At the same time, in the incident plane, it transmits for a certain distance along the interface direction, and then returns to the optical dense medium [17]. You et al. proposed a long-range surface plasmon resonance (SPR) mode, in which gold $(\mathrm{Au})$ is the excitation layer of SPPs, so as to improve the GH shift [18]. The sliver (Ag) film layer of SPR can easily adjust the position of the minimum reflection and the maximum GH shift [19]. The sensitivity of GH shift to the refractive index of the surrounding medium is obtained by optimizing the incident angle and the thickness of $\mathrm{Au}[20]$.

Compared with other metal oxides, Indium tin oxide (ITO) has the characteristics of anti-corrosion, high transmittance, good conductivity, etc., which are widely used in the field of optical sensing [21,22]. The GH shift of p-polarized laser beam reflected from ITO surface under complex field has been studied [23]. In addition, two-dimensional (2D) nanomaterial with high thermal conductivity, carrier mobility, wide band optical response spectrum and strong nonlinear optical properties have attracted more and more attention [24,25]. The 2D materials include graphene [26,27], black phosphorus (BP) [28,29], and transition metal dichalcogenides (TMDCs) [30,31]. Graphene is the most representative 2D material widely used in optical sensors [32]. Luo studied the electro-optical modulation and magneto-optical modulation of GH shift in the double graphene coated waveguide [33]. Zhou et al. researched the GH effect in graphene substrate system by transfer matrix method [34]. Zhao et al. theoretically proposed the GH shift of light beam in a defect photonic crystal composed of dielectric multilayer and graphene [35]. By optimizing the thickness and layers number of the $\mathrm{Au}-\mathrm{MoS}_{2}$-graphene hybrid, You et al. gained the highest GH shift of 235.8 [36]. Through the optimization and comparative analysis of the Au-ITO-TMDCs-graphene hybrid structure, Han et al. obtained the highest GH shift of 801.7 $\lambda$ with $\mathrm{MoSe}_{2}$ monolayer and graphene bilayer [37]. Zhu et al. discovered another promising 2D material, Blue phosphorene (BlueP) [38]. The BlueP has the same thermal stability as BP with a band width of $2 \mathrm{eV}$, so it has a broad application prospect for sensing applications [39]. In addition, because the monolayer of BlueP and TMDCs have the same hexagonal crystal structure, it is easy to construct the heterstructure of BlueP/TMDCs [40]. Srivastava obtained the SPR sensor of BlueP/MoS 2 heterostructure to improve the sensitivity [41]. Sharma et al. proposed to use the BlueP/TMDCs molybdenum disulfide heterostructure, and compared with the traditional graphene SPR sensors [42].

In this paper, the hybrid structure of BlueP/TMDCs (Blue/WS ${ }_{2}, \mathrm{BlueP} / \mathrm{MoS}_{2}, \mathrm{BlueP} / \mathrm{MoSe}{ }_{2}$, BlueP/ $/ \mathrm{WSe}_{2}$ ) and graphene coated with ITO and Ag thin film is proposed. The maximum GH shift achieved was $-2361 \lambda$ for four layers BlueP/ $/ \mathrm{WS}_{2}$ and monolayer graphene. In addition, the highest sensitivity for index sensing reached $2.767 \times 10^{7} \lambda / \mathrm{RIU}$, which is 5152.7 times higher than the traditional Ag structure. We believe that this scheme with 2D materials has potential in highly sensitive sensors.

\section{Design Consideration and Mathematical Model}

The hybrid structure of Ag-ITO-BlueP/TMDCs-graphene based on the Kretschmann structure is shown in Figure 1. The $p$-polarized He-Ne laser emitted at $632.8 \mathrm{~nm}$ is collimated by a Glan-Taylor prism. Under the Kretschmann structure, the glass slide coated with metal film is fixed on the base of equilateral prism made of high refractive index (RI) glass with refractive index matching solution [43]. The incident light is irradiated on the SPR sensor through the side of the equilateral triangular coupling prism. The prism coupling device is controlled by a mobile rotary table, so as to change the angle of the incident light.

In the following description of the refractive index (RI) in each layer, $\lambda$ is the wavelength of the incident light, and its unit is um. In the first layer, the SF11 prism with RI $\left(n_{1}\right)$ is obtained [43]:

$$
n_{1}=\sqrt{\frac{1.73759695 \lambda^{2}}{\lambda^{2}-0.013188707}+\frac{0.313747346 \lambda^{2}}{\lambda^{2}-0.0623068142}+\frac{1.89878101 \lambda^{2}}{\lambda^{2}-155.23629}+1}
$$


Then, in the second layer, BK7 glass with RI $\left(n_{2}\right)$ is obtained [44]:

$$
n_{2}=\sqrt{\frac{1.03961212 \lambda^{2}}{\lambda^{2}-0.00600069867}+\frac{0.231792344 \lambda^{2}}{\lambda^{2}-0.0200179144}+\frac{1.01046945 \lambda^{2}}{\lambda^{2}-103.560653}+1}
$$

The third layer is Ag thin film and its RI $\left(n_{3}\right)$ is obtained through the Drude model [45]:

$$
n_{3}=\sqrt{1-\frac{\gamma_{c} \lambda^{2}}{\gamma_{p}^{2}\left(\gamma_{c}+i \lambda\right)}}=\sqrt{1-\frac{17.6140 \lambda^{2}}{0.14541^{2} \times(17.6140+i \lambda)}}
$$

The ITO film as fourth layer with RI $\left(n_{4}\right)$ is [46]:

$$
n_{4}=\sqrt{3.8-\frac{\gamma_{c} \lambda^{2}}{\gamma_{p}^{2}\left(\gamma_{c}+i \lambda\right)}}=\sqrt{3.8-\frac{11.2107 \lambda^{2}}{0.56497^{2} \times(11.2107+i \lambda)}}
$$

Subsequently, the 2D material of BlueP/TMDCs and graphene with monolayer and RI is shown as Table $1[47,48]$.

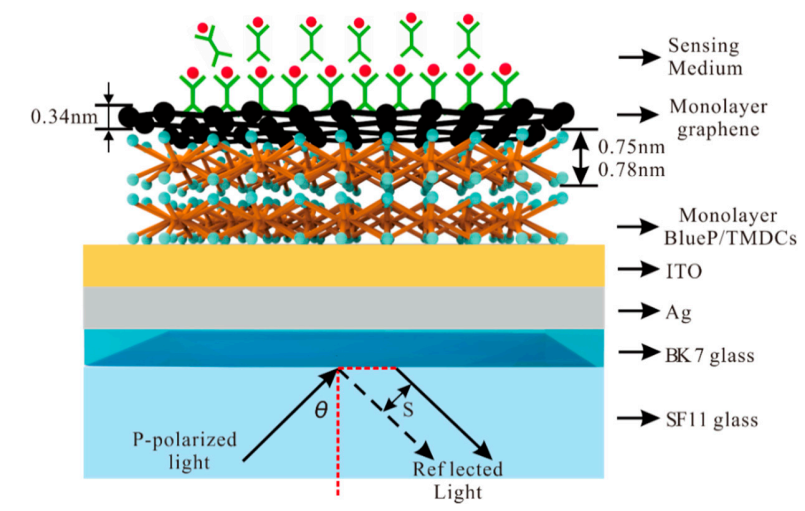

Figure 1. Schematic diagram of Ag- Indium tin oxide (ITO)-Blue Phosphorene (BlueP)/transition metal dichalogenides (TMDCs)-graphene enhanced Goos-Hänchen (GH) shift of surface plasmon resonance (SPR) sensor.

Table 1. The monolayer and refractive index (RI) of Blue Phosphorene (BlueP)/transition metal dichalogenides (TMDCs) and graphene at $\lambda=632.8 \mathrm{~nm}$.

\begin{tabular}{ccc}
\hline BlueP/TMDCs and Graphene & $\begin{array}{c}\text { Monolayer } \\
(\mathbf{n m})\end{array}$ & RI \\
\hline BlueP/WS $_{2}$ & 0.75 & $2.48+0.170 i$ \\
BlueP/MoS $_{2} / \mathrm{MoSe}_{2}$ & 0.75 & $2.81+0.320 i$ \\
BlueP/MoPse $_{2}$ & 0.78 & $2.77+0.350 i$ \\
BlueP/WSe $_{2}$ & 0.78 & $2.68+0.220 i$ \\
graphene & 0.34 & $3.00+1.149 i$ \\
\hline
\end{tabular}

The sensing medium is used for deionized (DI) water and its RI $\left(n_{7}\right)$ is obtained [43]:

$$
n_{7}=\sqrt{\sum_{i=1}^{4} \frac{A_{i} \lambda^{2}}{\lambda^{2}-t_{i}^{2}}+1}
$$

where the Sellmeier coefficients $A_{1}=0.5666959820, A_{2}=0.1731900098, A_{3}=0.02095951857, A_{4}=$ $0.1125228406, t_{1}=0.005084151894, t_{2}=0.01818488474, t_{3}=0.02625439472, t_{4}=10.73842352$. 
Therefore, the RI is $n_{1}=1.7786, n_{2}=1.5151, n_{3}=0.1350+3.9850 \mathrm{i}, n_{4}=1.858+0.058 \mathrm{i}, n_{7}=1.332+$ $n_{b i o}$. The $n_{b i o}$ represents the RI change of DI water. The thickness of BK7 glass and sensing medium is both $100 \mathrm{~nm}$. In order to compare the properties of 2D materials, we set the thickness of Ag and ITO to $45 \mathrm{~nm}$ and $10 \mathrm{~nm}$, respectively. When the number of graphene layers $\mathrm{n} \leq 5$, it is reasonable to treat monolayer as a non-interacting [49]. Hence, we only use graphene and BlueP/TMDCs with 5 layers or less, and ignore the interaction between them.

The thickness and dielectric constant of each layer are set as $d_{k}$ and $\varepsilon_{k}\left(\varepsilon_{k}=n_{k}^{2}\right)(k=1,2, \ldots 7)$. In order to analyze the reflectivity $\left(R_{p}\right)$ and phase $\left(\psi_{p}\right)$, the transfer matrix method (TMM) and the Fresnel equation based on $n$-layer model are used [47]. The SPR sensor is composed of parallel stacking in $\mathrm{Z}$ direction perpendicular to the sensing interface. The $M$ is the structure of the transmission matrix (TM), and $p$-polarized light is gained through the following relationship [43]:

$$
M=\prod_{k=2}^{N-1} M_{k}=\prod_{N}^{N-1}\left[\begin{array}{cc}
\cos \alpha_{k} & \left(-i \sin \alpha_{k}\right) / p_{k} \\
-i p_{k} \sin \alpha_{k} & \cos \alpha_{k}
\end{array}\right]=\left[\begin{array}{ll}
M_{11} & M_{12} \\
M_{21} & M_{22}
\end{array}\right]
$$

where

$$
p_{k}=\sqrt{\left(\frac{v_{k}}{\varepsilon_{k}}\right)} \cos \theta_{k}=\frac{\sqrt{\varepsilon_{k}-n_{1}^{2} \sin ^{2} \theta_{1}}}{\varepsilon_{k}}
$$

and

$$
\alpha_{k}=\frac{2 \pi d_{k}}{\lambda} \sqrt{\varepsilon_{k}-n_{1}^{2} \sin ^{2} \theta_{1}}
$$

The total reflection coefficient $\left(r_{p}\right)$ of $p$-polarized light is related to the matrix as follows:

$$
\gamma_{p}=\frac{\left(M_{11}+M_{12} p_{N}\right) p_{1}-\left(M_{21}+M_{22} p_{N}\right)}{\left(M_{11}+M_{12} p_{N}\right) p_{1}+\left(M_{21}+M_{22} p_{N}\right)}
$$

where $p_{1}$ corresponds to the SF11 prism layer and $p_{7}$ to the water layer. The $R_{p}$ and $\psi_{p}$ of the $p$-polarized light are shown as [17]:

$$
\begin{gathered}
R_{p}=\left|\gamma_{p}\right|^{2} \\
\psi_{p}=\arg \left(\gamma_{p}\right)
\end{gathered}
$$

We can use the fixed phase method to calculate the GH shift as followed:

$$
S=-\frac{1}{k_{0}} \frac{d \psi_{p}}{d \theta_{1}}=-\frac{\lambda}{2 \pi} \frac{d \psi_{p}}{d \theta_{1}}
$$

where the $\theta_{1}$ is the incident angle.

\section{Result and Discussion}

As shown in Figure 2, the reflectance, phase and GH shift of conventional Ag and Ag-ITO structure are compared and analyzed. The reflectivity (red dot line) and phase (blue dot line) are shown in Figure 2a. The SPR curve shows that there is a narrow reflection angle near $52.71^{\circ}$ and $55.22^{\circ}$ respectively, and the minimum reflectivity is 0.068 a.u for Ag structure and $0.014 \mathrm{a}$.u for Ag-ITO structure, respectively. In Figure $2 \mathrm{~b}$, the highest $\mathrm{GH}$ shift with $\mathrm{Ag}=45 \mathrm{~nm}$ is $60.21 \lambda$. With $\mathrm{Ag}=45 \mathrm{~nm}$ and ITO $=10 \mathrm{~nm}$, the maximum GH shift attains $62.11 \lambda$ indicating ITO can increase the GH shift and other performance.

Although ITO plays a certain role in the increase of GH shift, the enhancement is still relatively small. Next, we study the influence of the graphene layer, as shown in Figure 3. For the graphene monolayer, the reflectivity is 0.0024 a.u at $55.44^{\circ}$ and the $\mathrm{GH}$ shift is $125.7 \lambda$. When the graphene bilayer is added to the Ag-ITO structure, the best performance is obtained with the largest GH shift of $-439.7 \lambda$. 
We can observe that the phase change from Z-shaped-like to Lorentzian-like, and GH shift change from positive to negative. Then, with the increase of graphene layers, the GH shift is $-67.56 \lambda,-33.46 \lambda$, $-20.19 \lambda$, respectively.
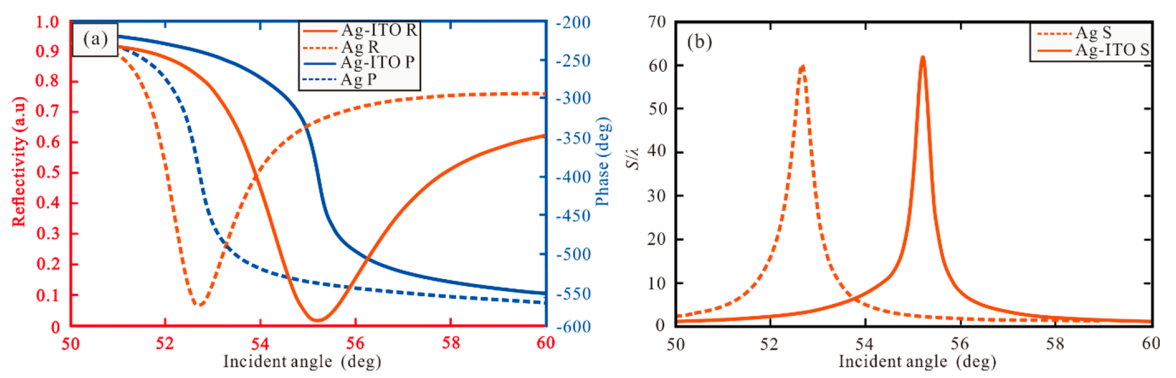

Figure 2. (a) Reflectivity and phase, and (b) GH shift for Ag and Ag-ITO structure with incident angle.

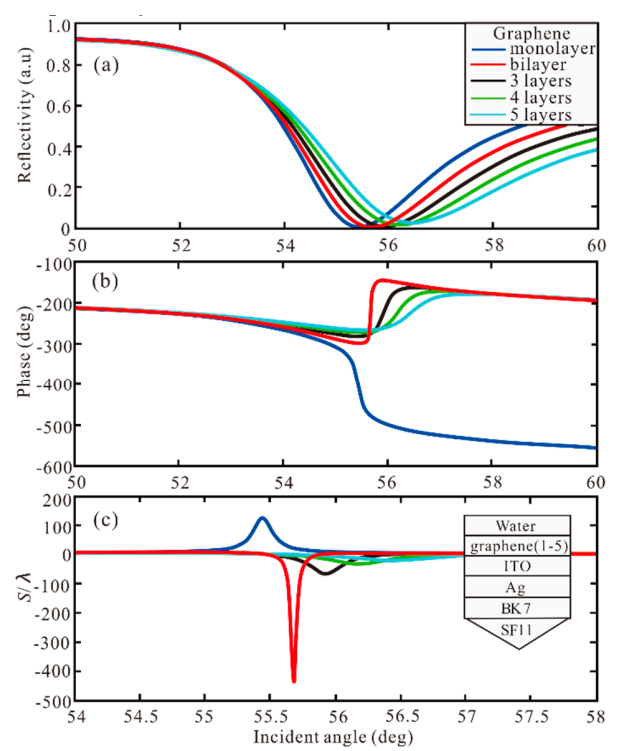

Figure 3. (a) Reflectivity, (b) Phase and (c) GH shift with incident angle for different number of graphene layers.

Similarly, the BlueP/TMDCs is added to the Ag-ITO structure, as shown in Figure 4. In Table 2, the optimal GH shift $(S / \lambda)$ with different number of BlueP/TMDCs is obtained. Hence, In the BlueP/TMDCs, we can understand that the BlueP/MoSe 2 has the greatest contribution to Ag-ITO structure.

Table 2. The optimal GH shift (S/ $\lambda$ ) with different number of BlueP/TMDCs.

\begin{tabular}{cccccc}
\hline BlueP/TMDCs & Monolayer & Bilayer & 3 Layers & 4 Layers & 5 Layers \\
\hline BlueP/MoS $_{2}$ & 77.31 & 111.6 & 241.2 & -662.2 & -139.5 \\
BlueP/WS $_{2}$ & 65.69 & 70.32 & 76.23 & 83.68 & 92.83 \\
BlueP/MoSe $_{2}$ & 82.42 & 141.5 & 1188 & -151.6 & -65.77 \\
BlueP/WSe $_{2}$ & 68.57 & 78.32 & 93.58 & 119.1 & 166.3 \\
\hline
\end{tabular}

From Figures 2-4, there are four important features. First, at a certain thickness of Ag-ITO film, due to the large real-part value of BlueP/TMDCs and graphene dielectric function, with the increase of BlueP/TMDCs and graphene layers, the SPR resonance angle has a large red shift. Second, the imaginary part of dielectric function of graphene layer is larger than that of BlueP/TMDCs layer, which leads to a large loss of electronic energy. Third, the resonance depth (i.e., the minimum reflectivity) strongly 
depends on the number of BlueP/TMDC and graphene layers deposited on Ag-ITO films. The light energy absorbed by Ag-ITO film is not enough to excite strong SPR. By further coating BlueP/TMDCs and graphene on the surface of Ag-ITO film, the light absorption of the hybrid structure can be enhanced effectively, thereby promoting a stronger SPR excitation.

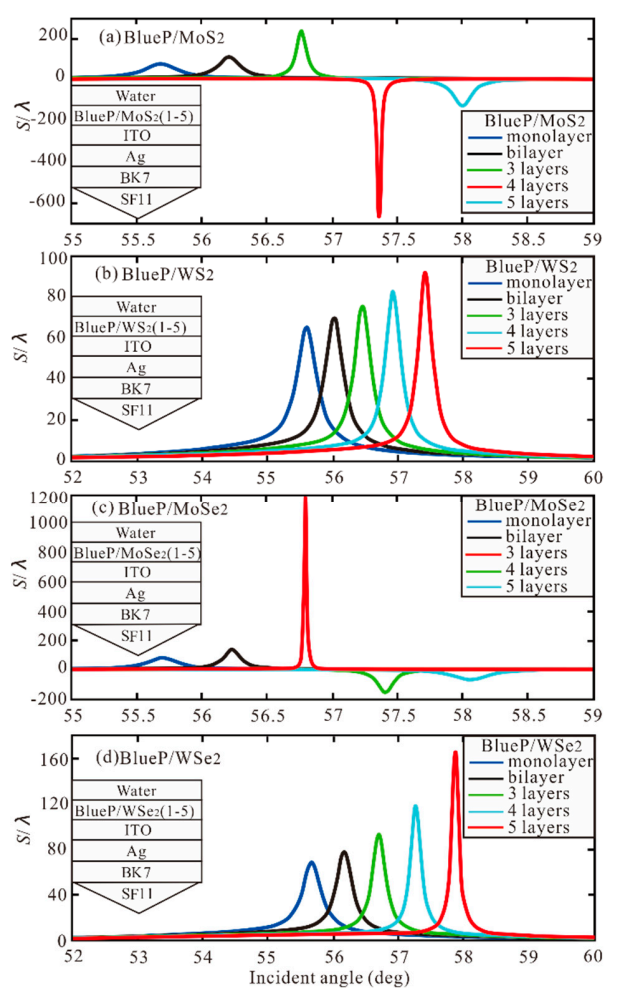

Figure 4. The GH shift with incident angle for different number of BlueP/TMDCs (a) BlueP/MoS 2 layers, (b) BlueP/WS 2 layers, (c) BlueP/MoSe 2 layers, (d) BlueP/WSe 2 layers.

Figure 5 shows the GH shift relative to the incident angle when graphene is monolayer and the number of BlueP/TMDCs layers changes from monolayer to five layers. The optimal GH shift and resonance angle with different number of BlueP/TMDCs and graphene monolayer are obtained as Table 3. We know that with the increase of the optimal GH shift of each BlueP/TMDCs, the corresponding resonance angle also increases.

Table 3. The optimal GH shift $(\mathrm{S} / \lambda)$ and resonance angle $(\theta)$ with different number of BlueP/TMDCs and graphene monolayer.

\begin{tabular}{cccc}
\hline \multirow{2}{*}{ BlueP/TMDCs } & \multicolumn{2}{c}{ Graphene (Monolayer) } \\
\cline { 3 - 4 } & & GH Shift $(\lambda)$ & Resonance Angle $(\boldsymbol{\theta})$ \\
\hline BlueP/MoS & bilayer & -385.8 & $56.48^{\circ}$ \\
BlueP/WS & 4 layers & -2361 & $57.23^{\circ}$ \\
BlueP/MoSe & monolayer & 456.9 & $55.96^{\circ}$ \\
BlueP/WSe & 3 layers & -665.5 & $56.99^{\circ}$ \\
\hline
\end{tabular}

Subsequently, the BlueP/TMDCs monolayer and different number of graphene layers are added to the Ag-ITO structure of SPR, as shown in Figure 6. The optimal GH shift with a different number of graphene and BlueP/TMDCs monolayer are obtained, as shown in Table 4. 


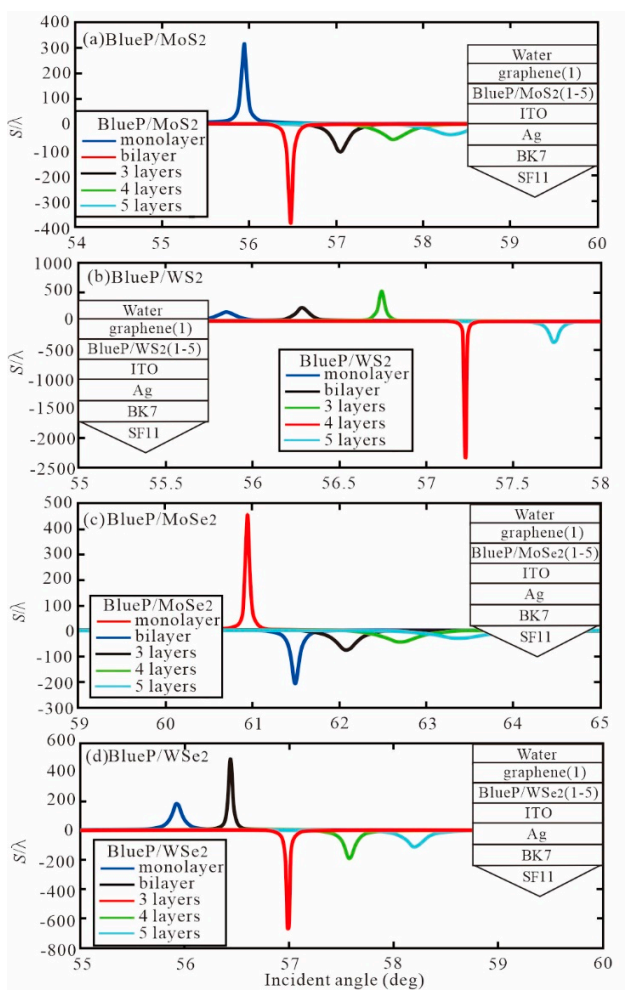

Figure 5. The GH shift with incident angle with graphene monolayer and different number of BlueP/TMDCs (a) BlueP/MoS 2 layers, (b) BlueP/WS 2 layers, (c) BlueP/MoSe 2 layers, (d) BlueP/ $\mathrm{WSe}_{2}$ layers.
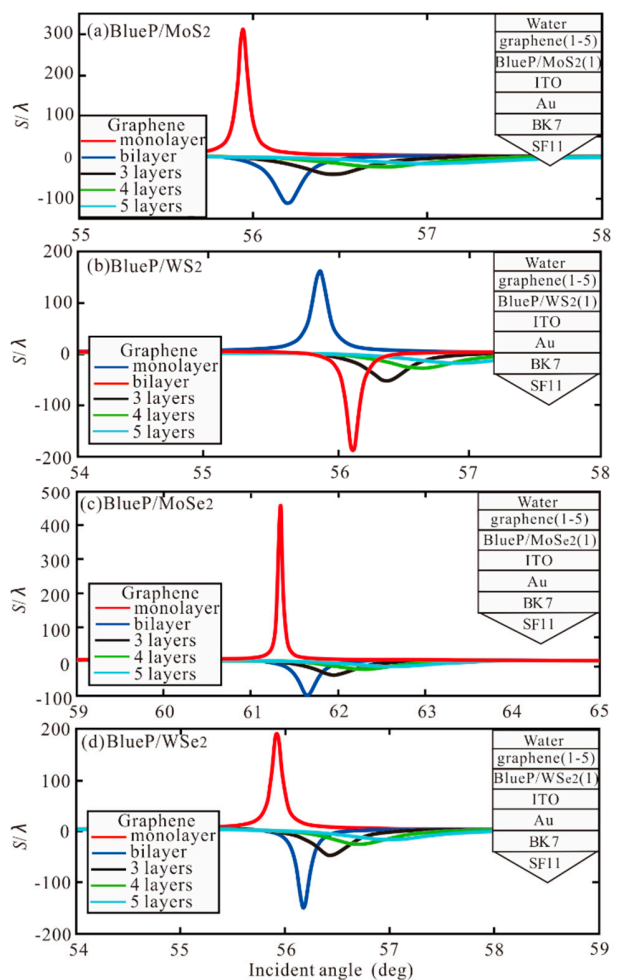

Figure 6. The GH shift with incident angle with BlueP/TMDCs monolayer and different number of graphene layers (a) BlueP/MoS 2 , (b) BlueP/WS $/ \mathrm{WS}_{2}$, (c) BlueP/MoSe 2 , (d) BlueP/WSe 2 . 
Table 4. The optimal GH shift $(S / \lambda)$ with BlueP/TMDCs monolayer and different number of graphene.

\begin{tabular}{cccccc}
\hline \multirow{2}{*}{ Graphene } & \multicolumn{3}{c}{ BlueP/TMDCs (Monolayer) } \\
\cline { 2 - 5 } & monolayer & BlueP/MoS $_{\mathbf{2}}$ & BlueP/WS $_{\mathbf{2}}$ & BlueP/MoSe $_{\mathbf{2}}$ & BlueP/WSe $_{\mathbf{2}}$ \\
\hline \multirow{3}{*}{ Graphene } & 315.6 & 164.1 & 456.9 & 193.1 \\
& bilayer & -112.2 & -189.3 & -205.1 & -153.6 \\
& 3 layers & -42.44 & -52.23 & -75.18 & -48.25 \\
& 4 layers & -24.35 & -27.95 & -43.27 & -26.49 \\
& 5 layers & -16.19 & -18.02 & -29.2 & -17.27 \\
\hline
\end{tabular}

In Table 5, the optimal GH shift with a different number of BlueP/TMDCs and graphene layers are summarized, where the bold indicates the highest $\mathrm{GH}$ shift value under the structure. In the BlueP/MoS 2 and graphene, the highest GH shift is $-385.8 \lambda$ in $\mathrm{BlueP} / \mathrm{MoS}_{2}$ bilayer and graphene monolayer. Then, the maximum GH shift with BlueP/ $\mathrm{WS}_{2}$ four layers and graphene monolayer is $-2361 \lambda$. Subsequently, the largest GH shift with BlueP/WSe $\mathrm{W}_{2}$ three layers and graphene monolayer of $-655.5 \lambda$ is obtained. Finally, the highest GH shift of $456.9 \lambda$ is obtained for both BlueP/MoSe 2 and graphene monolayer. Therefore, in the Ag-ITO-BlueP/TMDCs-graphene structure, BlueP/WS 2 has the greatest contribution to GH shift. The monolayer of graphene has the best performance.

Table 5. The optimal GH shift $(\mathrm{S} / \lambda)$ with different number of BlueP/TMDCs and graphene layers.

\begin{tabular}{ccccccc}
\hline \multirow{2}{*}{$\begin{array}{c}\text { Type of BlueP/TMDCs } \\
\text { and Graphene }\end{array}$} & \multicolumn{5}{c}{ Graphene } \\
\cline { 3 - 7 } & Monolayer & Bilayer & 3 Layers & 4 LAYERS & 5 layers \\
\hline BlueP/MoS $_{2}$ & bilayer & -385.8 & -59.85 & -29.7 & -18.58 & -12.9 \\
BlueP/WS $_{2}$ & 4 layers & -2361 & -64.34 & -29.65 & -18.09 & -12.4 \\
BlueP/WSe $_{2}$ & 3 layers & -665.5 & -60.99 & -29.16 & -18.01 & -12.43 \\
BlueP/MoSe $_{2}$ & monolayer & 456.9 & -205.1 & -75.18 & -43.27 & -29.2 \\
\hline
\end{tabular}

In our study, the RI of the sensing medium $\left(\Delta n_{7}\right)$ is changed, then and the GH shift is shown a giant red shift. Hence, the proposed new SPR heterostructure is used as a high sensitivity sensor based on shift variation. $\triangle G H$ is defined as highest value of the varying $G H$ shift and the sensitivity is defined as $S_{p}^{\prime}=\Delta G H / \Delta n_{7}$. We define the traditional SPR Ag, Ag-ITO, Ag-ITO-BlueP/WS 2 (monolayer), Ag-ITO-graphene (monolayer), Ag-ITO-BlueP/ $/ \mathrm{WS}_{2}$ (monolayer)-graphene (monolayer), $\mathrm{Ag}-\mathrm{ITO}-\mathrm{BlueP} / \mathrm{WS}_{2}$ (four layers)-graphene (monolayer) structure as Structure I to Structure VI and show as Figure 7. In Table 6, the optimal $S^{\prime}{ }_{P}$ with $\Delta G H$ and $\Delta n_{7}$ for Structure I to Structure VI are gained. Therefore, Structure VI is 5152.7 times higher than Structure I, 2470.5 times higher than Structure II, 2159.2 times higher than Structure III, and 688.9 times higher than Structure IV.

Table 6. The optimal $S_{P}^{\prime}$ with $\Delta G H$ and $\Delta n_{7}$ for Structure I to Structure VI.

\begin{tabular}{cccc}
\hline Structure & $\boldsymbol{\Delta G H}(\boldsymbol{\lambda})$ & $\boldsymbol{\Delta} \boldsymbol{n}_{\mathbf{7}}$ & $\boldsymbol{S}^{\prime} \boldsymbol{P}(\boldsymbol{\lambda} / \mathbf{R I U})$ \\
\hline Structure I & 10.74 & 0.002 & 5370 \\
Structure II & 22.4 & 0.002 & 11,200 \\
Structure III & 25.63 & 0.002 & 12,815 \\
Structure IV & 82.73 & 0.002 & 41,365 \\
Structure V & 125.17 & 0.002 & 62,585 \\
Structure VI & 2767 & 0.0001 & $2.767 \times 10^{7}$ \\
\hline
\end{tabular}



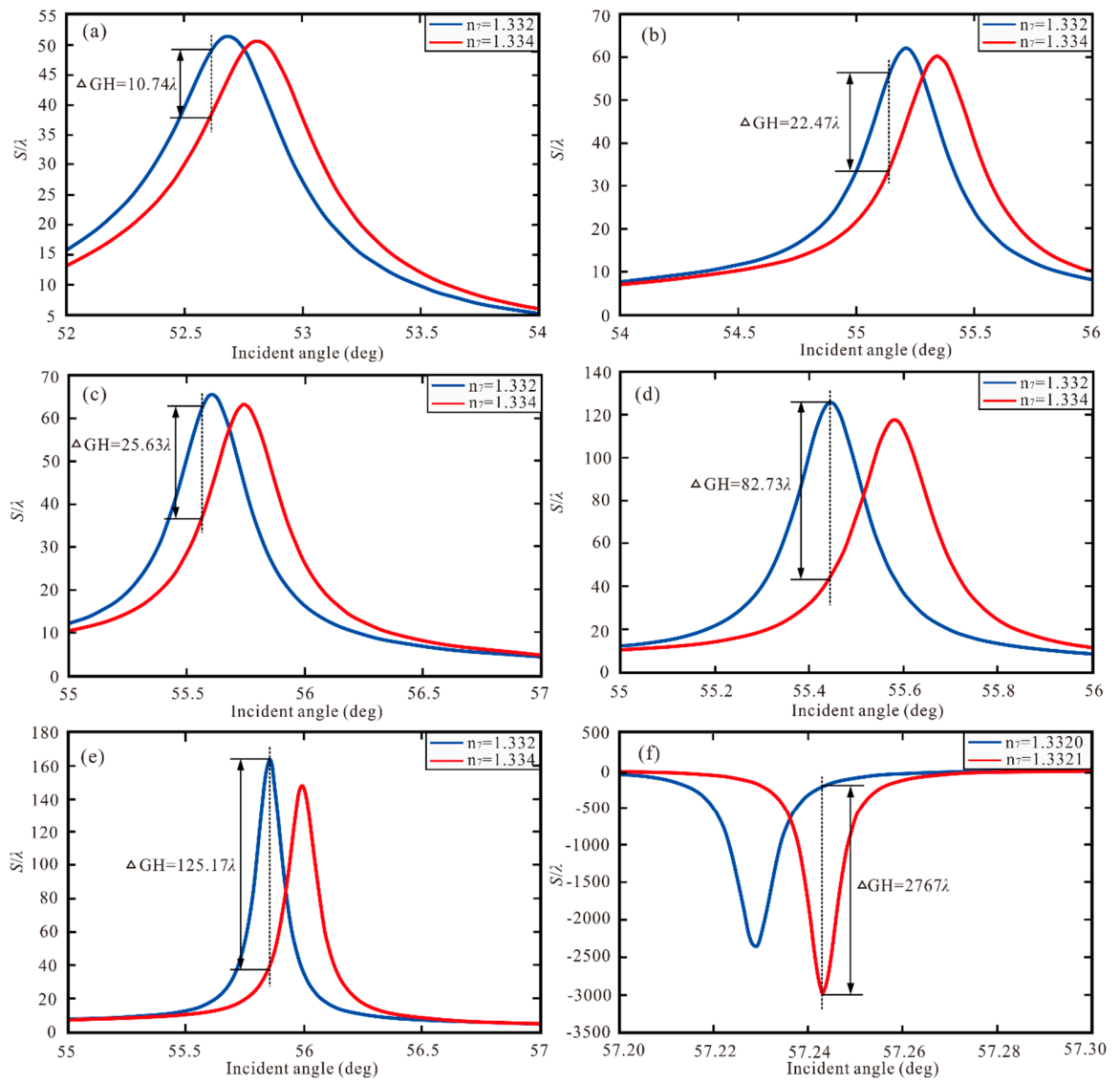

Figure 7. (a-e) GH shift with Structure I/Structure II/Structure III/Structure IV/Structure V with the $\Delta \mathrm{n}_{7}$ $=0.002,(\mathbf{f}) \mathrm{GH}$ shift with Structure VI with the $\Delta \mathrm{n}_{7}=0.0001$.

Better compared with previous research results, Table 7 summarizes the GH shift and sensitivity based on SPR sensor. In references [9], the GH shift of $12.5 \lambda$ is obtained by traditional Au thin film. In reference [50], when $\mathrm{MoS}_{2}$ of 2D material and air was added to the SPR biosensor, the GH shift was improved to $40.5 \lambda$. We can find that 2D material and air can improve the GH shift of SPR sensor. In reference [51], when the graphene replaced $\mathrm{MoS}_{2}$, the GH shift increased to 61.1 $\lambda$. Therefore, compared with $\mathrm{MoS}_{2}$, graphene improves the performance of the SPR sensor more significantly. However, when graphene and $\mathrm{MoS}_{2}$ were added to the $\mathrm{Au}$ film of SPR sensor, the GH shift increased to $235.8 \lambda$ for reference [36], and the highest sensitivity was obtained as $5.545 \times 10^{5} \lambda / \mathrm{RIU}$. In reference [37], when the ITO and $\mathrm{MoSe}_{2}$ replaced $\mathrm{MoS}_{2}$, the GH shift increased to $801.7 \lambda$, and the maximum sensitivity was $8.02 \times 10^{5} \lambda /$ RIU. In this work, we use BlueP/TMDCs instead of TMDCs, and change metal into Ag, so as to construct the SPR sensor with Ag-ITO- BlueP/ $\mathrm{WS}_{2}$-graphene hybrid structure. The optimal GH displacement is $2361 \lambda$ and the maximum sensitivity is $2.767 \times 10^{7} \lambda /$ RIU. Based on the analysis, we can see that our novel SPR sensor improves the GH shift and sensitivity significantly.

Table 7. Comparison with the previous studies GH shift and sensitivity SPR sensor.

\begin{tabular}{|c|c|c|c|}
\hline Structure & GH $\operatorname{shift}(\lambda)$ & Sensitivity( $\lambda /$ RIU) & References \\
\hline $\mathrm{Au}$ & 12.5 & - & [9] \\
\hline Air- $\mathrm{MoS}_{2}$ & 40.5 & - & [50] \\
\hline Air-graphene & 61.1 & - & [51] \\
\hline $\mathrm{Au}-\mathrm{MoS}_{2}$-graphene & 235.8 & $5.545 \times 10^{5}$ & [36] \\
\hline Au-ITO-MoSe ${ }_{2}$-graphene & 801.7 & $8.02 \times 10^{5}$ & [37] \\
\hline Ag-ITO-BlueP/WS ${ }_{2}$-graphene & 2361 & $2.767 \times 10^{7}$ & This work \\
\hline
\end{tabular}




\section{Conclusions}

In this paper, the GH shift of the Kretschmann configuration combined with SPR-based 2D nanomaterials is studied. When SPPs were excited, we theoretically proved the influence of the number of graphene and BlueP/TMDCs layers on the GH shift, and obtained a huge GH shift by using the mixed structure of BlueP/WS 2 four layers and graphene monolayer. The maximum $\mathrm{GH}$ shift is 2361 times that of the incident wavelength. Compared with the traditional SPR structure, the shift of the structure is increased by more than 39.21 times. In addition, by changing the number of BlueP/TMDCs layers, we can control the positive and negative shift of GH in the structure of BlueP/TMDCs-graphene. The maximum GH shift corresponding to the highest sensitivity is 2.767 $\times 10^{7} \lambda / \mathrm{RIU}$, which is 5152.7 times higher than the traditional SPR of Ag, 2462.8 times of Ag-ITO, 2159.2 times of Ag-ITO-BlueP/ $\mathrm{WS}_{2}$, and 688.9 times of Ag-ITO-graphene. The sensing layer we use is deionized water, therefore, it is suitable as a sensing medium with a refractive index close to 1.332 , to gain a higher sensitivity. This structure is expected to be a candidate for high-performance sensors.

Author Contributions: Conceptualization, L.H. and T.H.; methodology, L.H.; software, J.P.; validation, L.H.; formal analysis, D.L.; investigation, L.H.; resources, T.H.; data curation, Z.H.; writing—original draft preparation, L.H.; writing—review and editing, D.L.; visualization, J.P.; supervision, D.L.; project administration, D.L.; funding acquisition, T.H. All authors have read and agreed to the published version of the manuscript.

Funding: This research was funded by the National Key Technologies R\&D Program, grant number 2016YFC0201101, Natural Science Foundation of Hubei Province, grant number 2019CFB598, Wuhan Science and Technology Bureau, grant number 2018010401011297, National Natural Science Foundation of China, grant number 61876210, and the Fundamental Research Founds for Central University, China University of Geosciences (Wuhan), grant number 1910491B06.

Conflicts of Interest: The authors declare no conflict of interest.

\section{References}

1. Shaaban, A.; Hameed, M.F.O.; Gomaa, L.; Obayya, S.S.A. Accurate calculation of Goos-Hänchen shift at critical angle for complex laser beam profiles using beam propagation method. Optik 2018, 157, 1106-1114. [CrossRef]

2. Huamán, A.; Usaj, G. Anomalous Goos-Hänchen shift in the Floquet scattering of Dirac fermions. Phys. Rev. A 2019, 100, 033409. [CrossRef]

3. Li, S.; Wan, Y.; Liu, J.; Kong, W.; Zheng, Z. Effect of Excitation Beam Divergenceon the Goos-HänchenShift Enhanced byBloch Surface Waves. Appl. Sci. 2018, 9, 40. [CrossRef]

4. Li, K.; Cheng, F. Effects of strain on Goos-Hänchen shifts of monolayer phosphorene. Phys. E Low Dimens. Syst. Nanostruct. 2018, 97, 335-339. [CrossRef]

5. A Carvalho, S.; De Leo, S.; Huguenin, J.A.O.; Martino, M.; Da Silva, L. Experimental evidence of laser power oscillations induced by the relative Fresnel (Goos-Hänchen) phase. Laser Phys. Lett. 2019, 16, 065001. [CrossRef]

6. Jena, S.C.; Shrivastava, S.; Saxena, S.; Kumar, N.; Maiti, S.K.; Mishra, B.P.; Singh, R.K. Surface plasmon resonance immunosensor for label-free detection of BIRC5 biomarker in spontaneously occurring canine mammary tumours. Sci. Rep. 2019, 9, 1-12. [CrossRef]

7. Yu, W.J.; Sun, H.; Gao, L. Enhanced normal-incidence Goos-Hänchen effects induced by magnetic surface plasmons in magneto-optical metamaterials. Opt. Express 2018, 26, 3956-3973. [CrossRef]

8. Solookinejad, G.; Jabbari, M.; Nafar, M.; Sangachin, E.A. Enhancement of Goos-Hänchen shifts due to spontaneously generated coherence in a four-level Rydberg atom. Laser Phys. 2019, 29, 065203. [CrossRef]

9. Tang, T.; Li, J.; Luo, L.; Shen, J.; Li, C.; Qin, J.; Bi, L.; Hou, J. Weak measurement of magneto-optical Goos-Hänchen effect. Opt. Express 2019, 27, 17638-17647. [CrossRef]

10. Saito, H.; Neo, Y.; Matsumoto, T.; Tomita, M. Giant and highly reflective Goos-Hänchen shift in a metal-dielectric multilayer Fano structure. Opt. Express 2019, 27, 28629-28639. [CrossRef]

11. Malik, A.; Chaung, Y.-L.; Abbas, M. Ziauddin Giant negative and positive Goos-Hänchen shifts via Doppler broadening effect. Laser Phys. 2019, 29, 075201. [CrossRef] 
12. Stockschläder, P.; Kreismann, J.; Hentschel, M. Curvature dependence of semiclassical corrections to ray optics: How Goos-Hänchen shift and Fresnel filtering deviate from the planar case result. EPL Europhys. Lett. 2014, 107, 64001. [CrossRef]

13. Yin, X.; Hesselink, L.; Liu, Z.; Fang, N.X.; Zhang, X. Large positive and negative lateral optical beam displacements due to surface plasmon resonance. Appl. Phys. Lett. 2004, 85, 372-374. [CrossRef]

14. Yu, P.; Yang, H.; Chen, X.; Yi, Z.; Yao, W.; Chen, J.; Yi, Y.; Wu, P. Ultra-wideband solar absorber based on refractory titanium metal. Renew Energy 2020, 158, 227-235. [CrossRef]

15. Zhao, F.; Chen, X.; Yi, Z.; Qin, F.; Tang, Y.; Yao, W.; Zhou, Z.; Yi, Y. Study on the solar energy absorption of hybrid solar cells with trapezoid-pyramidal structure based PEDOT: PSS/c-Ge. Sol. Energy 2020, 204, 635-643. [CrossRef]

16. Li, J.; Shen, C.; Díaz-Rubio, A.; Tretyakov, S.A.; Cummer, S.A. Systematic design and experimental demonstration of bianisotropic metasurfaces for scattering-free manipulation of acoustic wavefronts. Nat. Commun. 2018, 9, 1342. [CrossRef]

17. Wei, L.; Picardi, M.F.; Kingsley-Smith, J.; Zayats, A.V.; Rodríguez-Fortuño, F.J. Directional scattering from particles under evanescent wave illumination: The role of reactive power. Opt. Lett. 2018, 43, 3393-3396. [CrossRef]

18. You, Q.; Zhu, J.; Guo, J.; Wu, L.-M.; Dai, X.; Xiang, Y.-J. Giant Goos-Hänchen shifts of waveguide coupled long-range surface plasmon resonance mode. Chin. Phys. B 2018, 27, 34-38. [CrossRef]

19. Kang, Y.; Gao, P.; Liu, H.; Zhang, J. Large Tunable Lateral Shift from Guided Wave Surface Plasmon Resonance. Plasmonics 2019, 14, 1289-1293. [CrossRef]

20. Guo, X.; Liu, X.; Zhu, W.; Gao, M.; Long, W.; Yu, J.; Zheng, H.; Guan, H.; Luo, Y.; Lu, H.; et al. Surface plasmon resonance enhanced Goos-Hänchen and Imbert-Fedorov shifts of Laguerre-Gaussian beams. Opt. Commun. 2019, 445, 5-9. [CrossRef]

21. Lian, J.; Zhang, D.; Hong, R.; Yan, T.; Lv, T.; Zhang, D. Broadband absorption tailoring of SiO2/Cu/ITO arrays based on hybrid coupled resonance mode. Nanomaterials 2019, 9, 852. [CrossRef] [PubMed]

22. Szunerits, S.; Castel, X.; Boukherroub, R. Surface Plasmon Resonance Investigation of Silver and Gold Films Coated with Thin Indium Tin Oxide Layers: Influence on Stability and Sensitivity. J. Phys. Chem. C 2008, 112, 15813-15817. [CrossRef]

23. Jiao, X.; Cai, Y.; Wang, Z. Goos-Hanchen and Imbert-Fedorov shifts of a laser beam reflected from ITO under complex fields. Opt. Commun. 2020, 457, 124712. [CrossRef]

24. Hlubina, P.; Urbancova, P.; Pudis, D.; Goraus, M.; Jandura, D.; Ciprian, D. Ultrahigh-sensitive plasmonic sensing of gas using a two-dimensional dielectric grating. Opt. Lett. 2019, 44, 5602-5605. [CrossRef]

25. Jia, A.-Q.; Sheng, M.-M.; Che, G.; Xu, C.; Zhang, Q.-F. Isolation and Structures of One- and Two-Dimensional High-Nuclearity Silver (I) Clusters from a Silver Propane-2-thiolate Chain. J. Clust. Sci. 2019, 1-8. [CrossRef]

26. Han, L.; Wu, C. A Phase Sensitivity-Enhanced Surface Plasmon Resonance Biosensor Based on ITO-Graphene Hybrid Structure. Plasmonics 2018, 14, 901-906. [CrossRef]

27. Maharana, P.K.; Srivastava, T.; Jha, R. On the Performance of Highly Sensitive and Accurate Graphene-on-Aluminum and Silicon-Based SPR Biosensor for Visible and Near Infrared. Plasmonics 2014, 9, 1113-1120. [CrossRef]

28. Wu, L.; Guo, J.; Wang, Q.; Lu, S.; Dai, X.; Xiangb, Y.; Fan, D. Sensitivity enhancement by using few-layer black phosphorus-graphene/TMDCs heterostructure in surface plasmon resonance biochemical sensor. Sens. Actuators B Chem. 2017, 249, 542-548. [CrossRef]

29. Srivastava, T.; Jha, R. Black Phosphorus: A New Platform for Gaseous Sensing Based on Surface Plasmon Resonance. IEEE Photon Technol. Lett. 2018, 30,319-322. [CrossRef]

30. Ouyang, Q.; Zeng, S.; Jiang, L.; Hong, L.; Xu, G.; Dinh, X.Q.; Qian, J.; He, S.; Qu, J.; Coquet, P.; et al. Sensitivity enhancement of transition metaldichalcogenides/silicon nanostructure-based surface plasmon resonancebiosensor. Sci. Rep. 2016, 6, 28190. [CrossRef]

31. Han, L.; Chen, Z.; Huang, T.; Ding, H.; Wu, C. Sensitivity Enhancement of Ag-ITO-TMDCs-Graphene Nanostructure Based on Surface Plasmon Resonance Biosensors. Plasmonics 2019, 15, 693-701. [CrossRef]

32. Fan, Y.; Tu, L.; Zhang, F.; Fu, Q.; Zhang, Z.; Wei, Z.; Li, H. Broadband Terahertz Absorption in Graphene-Embedded Photonic Crystals. Plasmonics 2017, 13, 1153-1158. [CrossRef] 
33. Luo, L.; Tang, T.; Shen, J.; Li, C. Electro-optic and magneto-optic modulations of Goos-Hänchen effect in double graphene coating waveguide with sensing applications. J. Magn. Magn. Mater. 2019, 491, 165524. [CrossRef]

34. Zhou, X.; Liu, S.; Ding, Y.; Min, L.; Luo, Z.; Luo, Z. Precise control of positive and negative Goos-Hänchen shifts in graphene. Carbon 2019, 149, 604-608. [CrossRef]

35. Zhao, N.; Ke, S.; Liu, Q.; Wang, B.; Lu, P. Giant Goos-Hänchen shifts in non-Hermitian dielectric multilayers incorporated with graphene. Opt. Express 2018, 26, 2817-2828. [CrossRef] [PubMed]

36. You, Q.; Shan, Y.; Gan, S.; Zhao, Y.; Dai, X.; Xiangb, Y. Giant and controllable Goos-Hänchen shifts based on surface plasmon resonance with graphene-MoS2 heterostructure. Opt. Mater. Express 2018, 8, 3036-3048. [CrossRef]

37. Han, L.; Pan, J.; Wu, C.; Li, K.; Ding, H.; Ji, Q.; Yang, M.; Wang, J.; Zhang, H.; Huang, T. Giant Goos-Hänchen Shifts in Au-ITO-TMDCs-Graphene Heterostructure and Its Potential for High Performance Sensor. Sensors 2020, 20, 1028. [CrossRef]

38. Zhu, Z.; Tománek, D. Semiconducting Layered Blue Phosphorus: A Computational Study. Phys. Rev. Lett. 2014, 112, 176802. [CrossRef]

39. Guan, J.; Zhu, Z.; Tománek, D. Phase Coexistence and Metal-Insulator Transition in Few-Layer Phosphorene: A Computational Study. Phys. Rev. Lett. 2014, 113, 046804. [CrossRef]

40. Peng, Q.; Wang, Z.; Sa, B.; Wu, B.; Sun, Z. Electronic structures and enhanced optical properties of blue phosphorene/transition metal dichalcogenides van der Waals heterostructures. Sci. Rep. 2016, 6, 31994. [CrossRef]

41. Srivastava, A.; Prajapati, Y.K. Performance Analysis of Silicon and Blue Phosphorene/MoS2 Hetero-Structure Based SPR Sensor. Photon Sens. 2019, 9, 284-292. [CrossRef]

42. Sharma, A.K.; Pandey, A.K. Blue Phosphorene/MoS2Heterostructure Based SPR Sensor with Enhanced Sensitivity. IEEE Photon Technol. Lett. 2018, 30, 595-598. [CrossRef]

43. Zeng, S.; Hu, S.; Xia, J.; Anderson, T.; Dinh, X.Q.; Meng, X.-M.; Coquet, P.; Yong, K. Graphene-MoS2 hybrid nanostructures enhanced surface plasmon resonance biosensors. Sens. Actuators B Chem. 2015, 207, 801-810. [CrossRef]

44. Han, L.; He, X.; Ge, L.; Huang, T.; Ding, H.; Wu, C. Comprehensive study of performance SPR biosensor based on metal-ITO-graphene /TMDCs hybrid multilayer. Plasmonics 2019, 14, 2021-2030. [CrossRef]

45. Gupta, B.D.; Sharma, A.K. Sensitivity evaluation of a multi-layered surface plasmon resonance-based fiber optic sensor: A theoretical study. Sens. Actuators B Chem. 2005, 107, 40-46. [CrossRef]

46. Han, L.; Ding, H.; Huang, T.; Wu, X.; Chen, B.; Ren, K.; Fu, S. Broadband Optical Reflection Modulator in Indium-Tin-Oxide-Filled Hybrid Plasmonic Waveguide with High Modulation Depth. Plasmonics 2017, 13, 1309-1314. [CrossRef]

47. Yue, C.; Lang, Y.; Zhou, X.; Liu, Q. Sensitivity enhancement of an SPR biosensor with a graphene and blue phosphorene/transition metal dichalcogenides hybrid nanostructure. Appl. Opt. 2019, 58, 9411-9420. [CrossRef]

48. Bruna, M.; Borini, S. Optical constants of graphene layers in the visible range. Appl. Phys. Lett. 2009, 94, 31901. [CrossRef]

49. Gan, C.H. Analysis of surface plasmon excitation at terahertz frequencies with highly doped graphene sheets via attenuated total reflection. Appl. Phys. Lett. 2012, 101, 111609. [CrossRef]

50. Das, A.; Pradhan, M. Goos-Hänchen shift for Gaussian beams impinging on monolayer-MoS2-coated surfaces. J. Opt. Soc. Am. B 2018, 35, 1956-1962. [CrossRef]

51. Zheng, Z.; Lu, F.; Jiang, L.; Jin, X.; Dai, X.; Xiang, Y. Enhanced and controllable Goos-Hänchen shift with graphene surface plasmon in the terahertz regime. Opt. Commun. 2019, 452, 227-232. [CrossRef]

(C) 2020 by the authors. Licensee MDPI, Basel, Switzerland. This article is an open access article distributed under the terms and conditions of the Creative Commons Attribution (CC BY) license (http://creativecommons.org/licenses/by/4.0/). 